\title{
An ECC Based Multibiometric System for Enhancing Security
}

\author{
U. Mahalakshmi ${ }^{1 *}$ and V. S. Shankar Sriram² \\ 'PG Student, School of Computing (CSE), SASTRA University, 613401, Thanjavur, \\ Tamilnadu, India; u.r.mahalakshmi@gmail.com \\ ${ }^{2}$ Associate Professor, School of Computing (IT), SASTRA University, 613401, Thanjavur, \\ Tamilnadu, India; sriram.it@sastra.edu
}

\begin{abstract}
Authentication schemes employing multibiometric system begin with several identification and verification based security schemes. Current systems implementing multi biometric scheme remain unsatisfactory facing to the growing security needs. The proposed system provides secured authentication to integrate multi biometric system with Elliptic Curve Cryptography that employs two modalities (i) Fusion of fingerprint, face, iris and signature. (ii) Generating elliptic curve and key using Elliptic Curve Cryptography. In the proposed system, selected portion of multi biometric features are fused into a single image and a curve is generated using ECC based technique that employs secured domain parameters generated through Genetic Algorithm. A one time password is also appended to the system to afford high authentication. The proposed algorithm is highly efficient against False Acceptance Rate and False Rejection Rate.
\end{abstract}

Keywords: Multibiometric, Elliptic Curve Cryptography, One time password, Authentication.

\section{Introduction}

Information security is the method of protecting information from unauthorized access, use, revealing, perturbation, alteration, perusal, scrutiny, and demolition. It is a common word that can be used despite of the form the data could take. Authentication plays a major role in security. Security awareness is most important in today's world. People are observing for a new way to the security that should be more reliable and authentic.

Biometric is a method of establishing the recognition of a person using personal or behavioural features. Biometric traits like face, finger print, iris, ears, vein, palm, voice etc are used as features. It is used for secure identification and verification. These are used in confidential financial transactions and personal data privacy. There are three most important modalities of biometric families [1]. Those are Biological, Behavioural and Morphological.
In Biological modality, it focuses on an individual biological data analysis. E.g. DNA analysis, heart beat etc. In Behavioural, it concentrates on an individual behavioural analysis while he is carrying out a particular task. E.g. way of walking, way of handling mouse etc and in morphological, it focuses on various different physical patterns. E.g. recognizing face, fingerprint etc...

Several trade applications depend on biometrics. Biometrics is the only method of assuring the existence of the owner when a transaction is done. The majority of the recent biometric applications make use of simply single biometric trait.

Figure 1 represents the unibiometric traits and systems that use these data are referred as unibiometric systems. But a unibiometric system has some limitations. This type of system depends on single biometric feature. It does not have sufficient information or favouritism. There are still questions that contrastingly manipulate their performance

* Corresponding author:

U. Mahalakshmi (u.r.mahalakshmi@gmail.com) 
such as unwanted data, limited degree of freedom, spoof attack and error rates. So, this paper concentrates on multibiometrics. Multibiometric is a combination of two or more unibiometric traits like finger print, iris, face and signature. These systems are capable of eradicating various drawbacks of the unibiometric systems by integrating the multiple sources of information. It is done by fusing various traits of a person, or multiple feature extraction on the same biometric.

Figure 2 represents the multibiometric traits. Also these systems know how to afford higher realisation efficiency on greater population coverage. However, a multibiometric system desires security for the reposting of multiple templates of each individual. Cryptosystems likes this focuses on encrypting the multiple templates (finger print, iris, face, signature) of each user in order to meliorate the consistency and efficiency of the biometric systems.

\subsection{Advantages of Multibiometrics}

1. Multibiometrics system deal with the problem of non-universality. If a user's dry finger averts her from favourable enrolling into a fingerprint system, then the next biometric trait such as iris may help in the admittance of the user [2]. For authentication based applications a subset of these traits are used under the deliberation and availability of the user.

2. It is complicated for a pretender to burlesque multiple traits of an individual that is legally enrolled or registered.
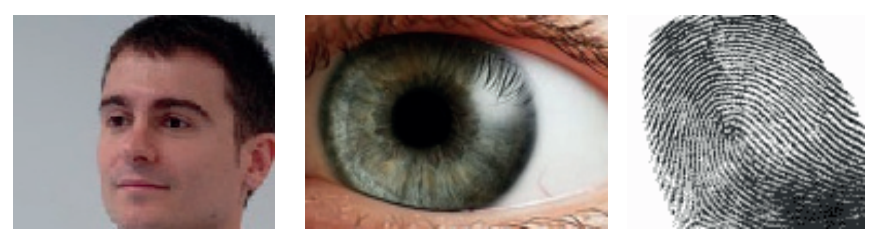

Figure 1. Unibiometric traits.

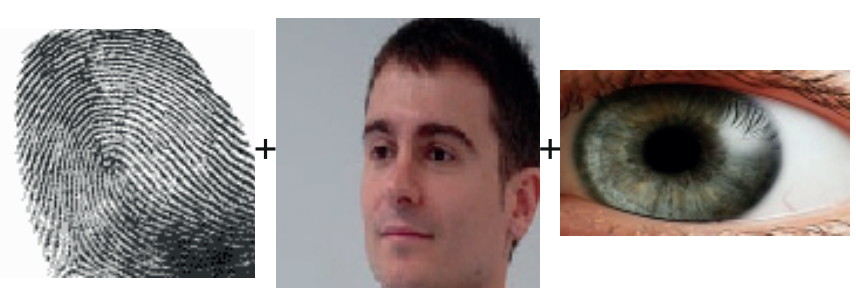

Figure 2. Multibiometric traits (Combination of three unibiometric traits).
3. It assists in continuous supervising and following of an individual in some condition when a single feature is not enough.

4. A Multibiometric system may perhaps be observed as fault tolerant system [2]. Owing to sensor or malfunction of software, some biometrics sources turn into defective.

The rest of the paper addresses the following: Section 2 concentrates on the previous work, section 3 explains the proposed system, section 4 examines the results and discussion and section 5 concludes this paper.

\section{Related Work}

Nagar et al. [3] proposed the feature level fusion of multibiometric templates. For higher level security, the multiple traits of an individual are combined into a single secure sketch. There are three phases in this paper. First phase is to obtain biometric characteristics and convert to binary string, and in second phase is to combining the above biometric traits and third phase is securely sketching. Fuzzy vault and fuzzy commitments are algorithms used in this paper for decoding. The former uses a Berlekamp-massae algorithm and latter decoding depends on the crossover probabilities.

Juels et al. [4] proposed fuzzy commitment scheme for providing authentication for biometric systems. It is used in biometric data for error tolerance. It converts the data into hash functions and stores the data in a server. This deals with the leading problems in authentication of biometric systems.

Yau [5] proposed classifier fusion problem which is the process of merging fingerprint and speech biometric decisions. They suggest constructing the various combinations of hyperbolic functions by network model. The suggested hyperbolic function is to demonstrate the approximation capability. At last it is exercised to combining the fingerprint and speech identification and verification to generate the best results.

Fu et al. [6] proposed a method of multibiometric cryptosystem, by binding the multiple features of biometrics to cryptography. There are two levels of combining, i.e. combining at the biometric level and combining at the cryptographic level. Shannon entropy is used to afford security. Accuracy and efficiency are also evaluated and it was compared with other systems.

Zhang et al. [7] proposed an encryption scheme and authentication scheme. This scheme is referred as mSEAS. 
The authentication and encryption scheme is based on the multibiometric data, with the intention of considering the privacy, unforgeability, and authentication. In addition it establishes the fuzzy extractor algorithm. By means of biometric string reader the information is excerpted. This can be used in environment of biometric authentication.

Jain [8] observe the various types of score normalization technique. Hand geometry, fingerprint, face traits were used in this paper for authentication. Normalization techniques like $\mathrm{z}$-score, min-max, tanh methods were used. This performs better, strong and efficient when compared to other systems.

Sumathi [9] proposed the multibiometric authentication using Discrete wavelet transform (DWT). A new novel technique based on DWT for identification of user. It utilizes support vector machine for the absolute result. The efficiency of the system is analysed in terms of False Acceptance Rate and Genuine Acceptance Rate.

Veeramachaneni [10] proposed an adaptive multimodal biometric management algorithm for multimodal biometric. It is a developing approach that moves towards the biometric security of sensor management. It is adaptive because, depends on the user requirement it alters in time. To make use of the best results in the system performance it selects the fusion rule. It also uses the sensor operating points.

Though extensive research is going on in the field of multibiometric authentication, so far no attempts has been made to generate one time password based on an individual biometric traits. This research contribution attempts to address this lacuna for generating a one time password using multibiomtric cryptosystem.

\section{Proposed Work}

Secured authentication is based on multibiometric cryptosystems. For multibiometric, the various traits of an individual are used. Elliptic curve cryptography (ECC) technique is used to generate curve and key. For providing a secured authentication this paper incorporates the use of one time password (OTP). The proposed system can be applied in financial based application services.

Figure 3 shows the system architecture. When a user provides his multibiometric traits the images are resized and fused into a single image. A matrix is generated using fixed points from the fused image, elliptic curve and key is generated using parameters $p, a, b$. Overlap the curve with the fused image and generate the one time password

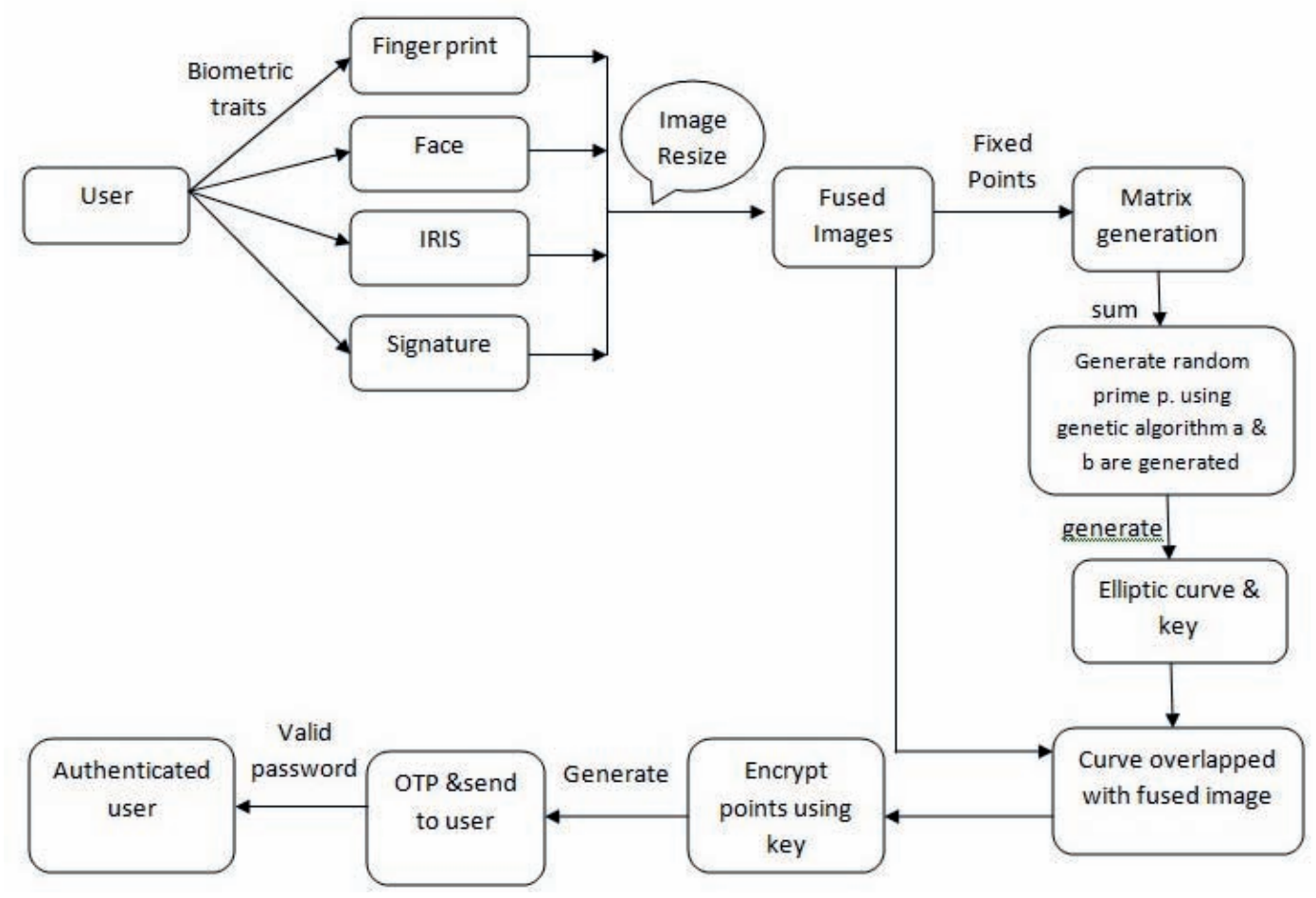

Figure 3. System Architecture. 
by encrypting the points using the key, which is send to user mobile. If it is valid password, then the user is authenticated.

\subsection{Fusion of Traits}

Multibiometric means combination of two or more traits. These systems are used mainly for authentication purpose. First get the input traits like fingerprint, face, iris and signature from individuals. To overcome the attacks from fraudsters, in Figure 4 the proposed system focuses mainly on combining these images (fingerprint, face, iris, and signature) into a single image, resize all the images to the fixed size and store in database.

\subsection{Generating Elliptic Curve and Key}

An elliptic curve is a non-singular, algebraic variety curve. ECC is a public key cryptography and in cryptosystem it provides greater flexibility. The key sizes are smaller when compared to other systems. Figure 5 represents the elliptic curve.

The elliptic equation is of the form:

$$
\begin{gathered}
y^{2}=x^{3}+a x+b \\
4 a^{3}+27 b^{2} \neq 0 \\
x, y, a, b \in R
\end{gathered}
$$

For generating curve, the elliptic curve method is applied and parameters such as $\mathrm{p}, \mathrm{a}, \mathrm{b}$ are used. To generate a novel curve a prime $p$ is needed. The upper and lower bound of curves are achieved by varying $\mathrm{a}$ and $\mathrm{b}$ from the Hasse Bound. To form an elliptic curve, take some 8 points from the fused single image. Obtain the pixel values from that 8 point, the ARGB (Alpha, Red, Green, Blue) colour model. Construct a matrix of size $n^{\star} n$ with these values, count the values in the matrix row by row, and sort the resultant values in the ascending order. Find the minimum value from the resultant values and generate a random prime number. The parameter $\mathrm{p}$ value is obtained and a, b may take any values from 0 to $\mathrm{p}-1$, but it should not abuse the non-singularity restraint i.e.

$$
4 a^{3}+27 b^{2} \neq 0
$$

For the corresponding prime $\mathrm{p}$, generate $\mathrm{a}, \mathrm{b}$ values using genetic algorithm.

\subsubsection{Genetic Algorithm}

Following are the steps of working genetic algorithm. Readers may refer to [13] for details on the working of genetic algorithms.

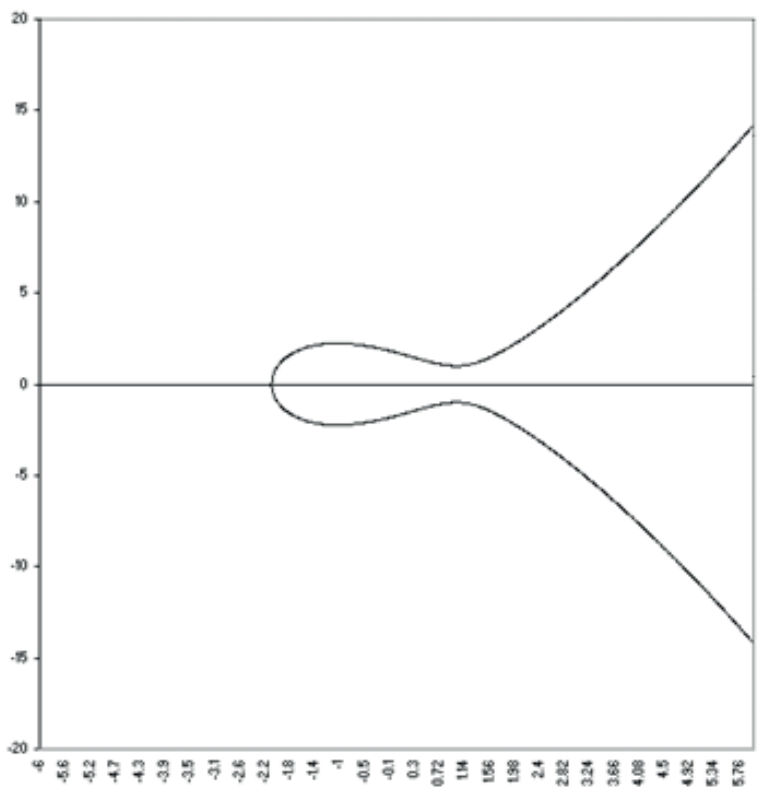

Figure 5. Elliptic curve.

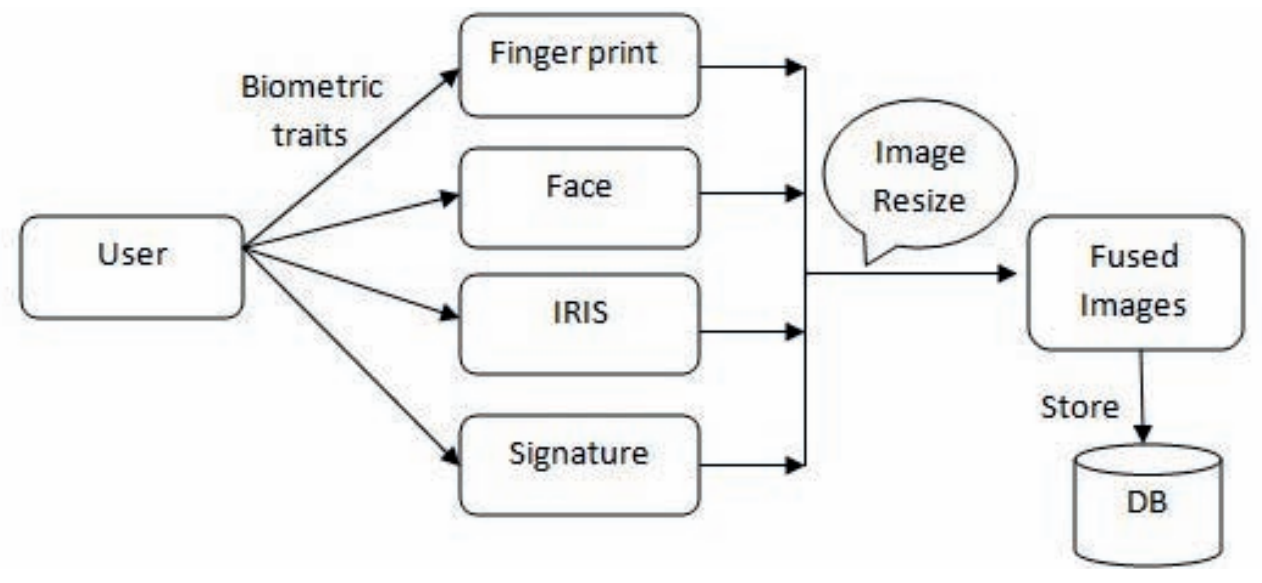

Figure 4. Fusion of traits. 
1. Randomly initialize a population of genes.

2. Divide the entire solution space into a fixed number of equal-sized chunks and apply steps $3-8$ to each chunk.

3. Scale the fitness of each gene in the population.

4. Perform reproduction on the population by applying a suitable selection operator.

5. Mutate the genes with a specified probability.

6. Apply crossover to the population to obtain a new pool of genes.

7. Perform elitism in order to retain the fittest genes of the previous population.

8. If the required number of generations has not been completed, return to step 3 .

9. If more unsearched chunks of the solution space remain, pick a new chunk and return to step 3 .

10. Find globally best gene among all chunks.

Table.1 shows the experimental setup results and Figure 6 represent the comparison of genes. The experimental results prove that the genetic algorithm successfully converges to highly fit solutions.

To determine the highest order of curve, make use of the existing algorithm called Schoof's algorithm. After successful generation of elliptic curve parameters, a key need to be generated using elliptic curve Diffie-Hellman (ECDH).

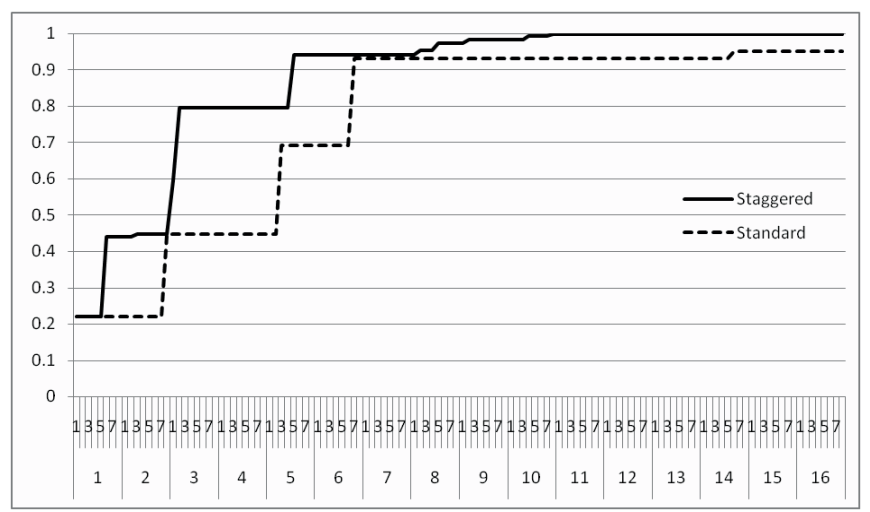

Figure 6. Comparison of performance when using staggered representation of genes vs. s standard representation of genes. Experimental Setup 2 - Run 2 was used.

A secret key is generated by using key specification. Intended readers may refer $[11,12]$ for more information on ECC.

\subsection{One Time Password (OTP)}

An OTP is legitimate for only one login session. It will be sent directly to the user's mobile phone. It is a dynamic password that will differ for each and every transaction and mainly used for authentication based services. Overlap the elliptic curve generated with the fused image, encrypt the

Table 1. Showing the experimental setup used in obtaining the results

\begin{tabular}{|c|c|c|c|}
\hline & & \multicolumn{2}{|c|}{ Experimental Setup } \\
\hline 1 & & \multicolumn{2}{|c|}{64} \\
\hline $\mathrm{n}$ & & \multicolumn{2}{|c|}{16} \\
\hline $\mathrm{k}$ & & \multicolumn{2}{|c|}{128} \\
\hline $\mathrm{d}$ & & \multicolumn{2}{|c|}{16} \\
\hline g & & \multicolumn{2}{|c|}{8} \\
\hline c & & \multicolumn{2}{|c|}{2.0} \\
\hline PM & & \multicolumn{2}{|c|}{0.02} \\
\hline \multirow[t]{3}{*}{$\mathrm{e}$} & & \multicolumn{2}{|c|}{12.5} \\
\hline & & Run 1 & Run 2 \\
\hline & $\mathrm{p}$ & 10780890715661305571 & 16819977131651944669 \\
\hline \multirow[t]{2}{*}{$\# \mathrm{E}(\mathrm{a}, \mathrm{b})$} & Lower Bound & 10780890709094452227 & 16819977123449511585 \\
\hline & Upper Bound & 10780890722228158916 & 16819977139854377754 \\
\hline \multirow[t]{4}{*}{ Solution } & $\mathrm{a}$ & 7540064068348742995 & 8156141376803454407 \\
\hline & $\mathrm{b}$ & 7972838799391103177 & 7525910221990009172 \\
\hline & Fitness & 0.994991 & 0.997302 \\
\hline & $\# \mathrm{E}(\mathrm{a}, \mathrm{b})$ & 10780890722162369732 & 16819977139810114186 \\
\hline
\end{tabular}


points that touches the curve using the generated secret key and send as OTP.

\subsection{Multibiometric Based Authentication using ECC (Proposed Algorithm)}

1. Get the input traits like fingerprint, iris, face, signature from the user.

2. Resize all the images to fixed size.

3. Combined these four images into a single fused image.

4. Select fixed (say 8) points on the fused image and form a matrix with pixel values of the colour model ARGB.

5. Form a matrix of size $n^{\star} n$.

6. Count the values in the matrix row by row and sort the resultant values in the ascending order.

7. Find the minimum value and generate a random prime $\mathrm{p}$.

8. Generate $a, b$ values using genetic algorithm for the corresponding prime $\mathrm{p}$.

9. Generate the elliptic curve and ECC key using ECC parameters ( $p, a, b)$.

10. Overlap the elliptic curve with the fused image and determine the points.

11. Encrypt the points using the ECC secret key and generate OTP.

\section{Results and Discussion}

This section discusses the performance analysis of proposed system and existing system. In the proposed system the multibiometric data are taken from the CASIA database. The proposed system is implemented in .NET framework visual studio 2008, database used is MS-SQL server 2005 and windows 7 operating system is used. For measuring the performance of the multibiometric system, False Acceptance Rate (FAR) and False Rejection Rate (FRR) are calculated.

$$
\begin{gathered}
\text { FAR }=\frac{\text { No. Of successful fraud attempts }}{\text { Total No. Of fraud attempts }} \\
\text { FRR }=\frac{\text { No. Of rejected verification attempts }}{\text { Total No. Of verification attempts }}
\end{gathered}
$$

This Figure 7 represents the FRR and FAR count of existing and proposed. Different cases are included, each describes the lower count of FAR and FRR than the existing multibiometric system.

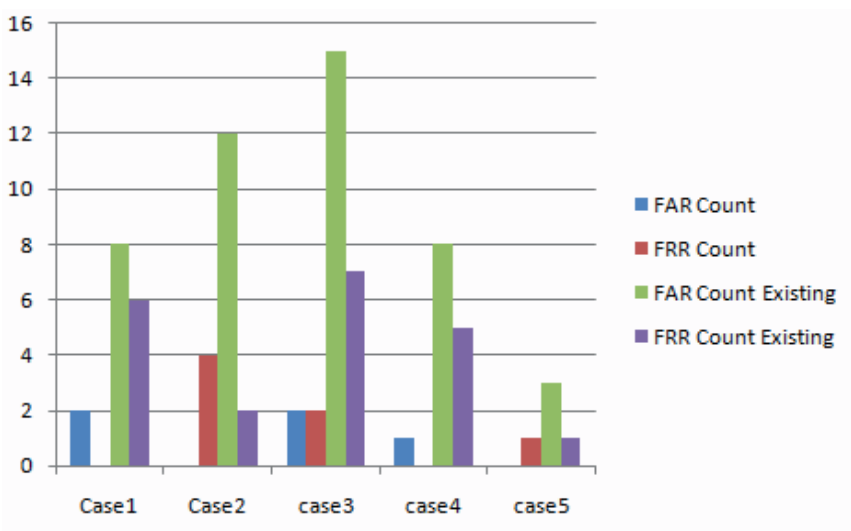

Figure 7. FAR and FRR count.

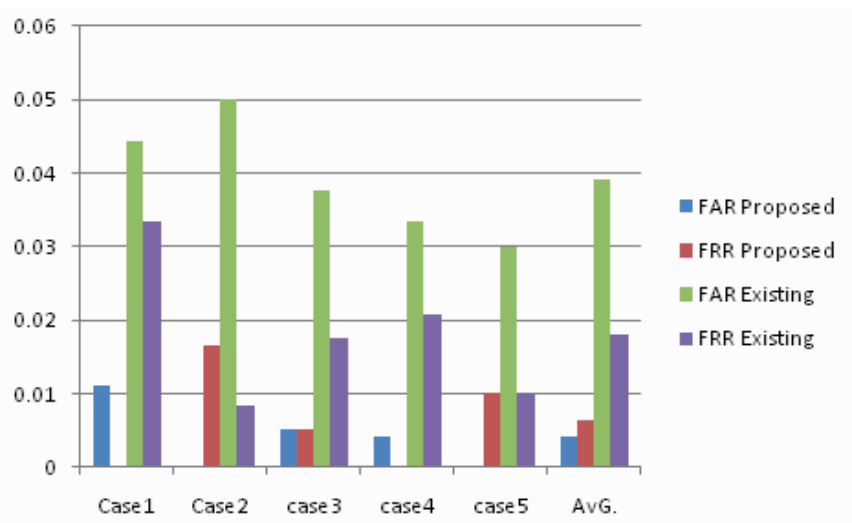

Figure 8. Performance evaluation.

This Figure 8 represents the evaluation of FAR and FRR. Different cases are tested and the performance is based on 200 trained data, 300 trained data, 500 trained data 240 and 125 trained data. Test data's are 180, 240, 398, 240 and 100 respectively. FAR is 0.01 and FRR is zero in casel. In test case 2 FAR is zero and FRR is 0.01 , in case 3 FAR is 0.05 and FRR is 0.05 , in test case 4 FAR is 0.01 and zero and in test case 5 FAR is zero and FRR is 0.01 .And the last is the average of all the test cases. From the above evaluation, by increasing the test data, FAR and FRR decreases as compared to the existing biometric systems.

\section{Conclusion}

This paper has provided overview of multibiometric systems. In the proposed system a novel approach has been used in multiple traits of the user are fused and stored in database. This system implements the ECC and OTP methodologies to maintain a high level authentication. This system improves the accuracy and efficiency rate in authentication. 


\section{References}

1. Giot R, and Rosenberger C (2012) .Genetic programming for multibiometrics, Expert Systems with Applications, vol 39(2), 1837-1847.

2. Ross A (2007) An Introduction to Multibiometrics, Proceedings of the $15^{\text {th }}$ European Signal Processing Conference (Poznam, Poland).

3. Nagar A, Nandhakumar K et al. (2012). Multibiometric cryptosystem based on feature level fusion, IEEE Transaction on Information Forensics and Security, vol 7(1), 255-268.

4. Juels A, and Wattenberg M (1999). A fuzzy commitment scheme, Proceedings of the Sixth ACM Conference On Computer and Communications Security, Singapore, 28-36.

5. Yau W (2004). Combination of hyperbolic functions for multimodal biometrics data fusion, IEEE Transaction on System, Man, Cybernetics, vol 34(2), 1196-1209.

6. Fu B, Yang S X et al. (2009) Multibiometric cryptosystem: Model structure and performance analysis, IEEE Transactions on Information Forensics Security, vol 4(4), $867-882$.
7. Zhang M, Yang B et al. (2011) Multibiometric based secure encryption and authentication scheme with fuzzy extractor, International Journal of Network Security, vol 12(1), 50-57.

8. Jain A, Nandakumar K et al. (2005) Score normalization in multimodal biometric systems, The Journal of Pattern Recognition Society, vol 38(12), 2270-2285.

9. Sumathi S, Hemamalini R (2012). Multibiometric authentication using DWT and score level fusion, European Journal of Scientific Research, vol.80(2), 213-223.

10. Veeramachaneni K, Osadciw L A et al. (2005). An adaptive multimodal biometric management algorithm, IEEE Transactions on systems, Man, and Cybernetics-Part C: Applications and Reviews, vol 35(3), 344-356.

11. SEC 1: Elliptic Curve Cryptography (2000), Certicom Research, Available from http://www.secg.org/collateral/ sec1_final.pdf, 1-90.

12. Hankerson D, Menezes A et al. (2004). Guide to elliptic curve cryptography, Chapter 3, Springer, 75-95.

13. Miller B L, Goldberg D E (1996). Genetic algorithms, selection schemes, and the varying effects of noise, Evolutionary Computation, vol 4(2), 113-131. 